\title{
Antibody-free method for protein detection on blots using enzyme fragment complementation
}

\author{
Joe Horecka, Neil W. Charter, Betty L. Bosano, Peter Fung, Phil Kobel, \\ Kun Peng, and Richard M. Eglen
}

BioTechniques 40:381-383 (March 2006)

doi 10.2144/000112119

Characterization of protein expression in recombinant systems has been enhanced through the availability of techniques such as Western blot analysis and enzyme-linked immunosorbent assay (ELISA) (1). A limitation of these techniques is the need for an antibody specific to the protein of interest. The development of epitope tags such as c-myc and HA has addressed this limitation by providing the means to characterize recombinant proteins without the need for a protein-specific antibody $(2,3)$. While they provide good utility, techniques such as Western blotting involve multiple incubation and wash steps that require a significant amount of time to perform. Thus, there exists a need for a more efficient method to detect and characterize recombinant proteins. We have developed an alternative blot assay, which provides similar utility to Western blotting without the need for specific antibodies or multiple incubation and wash steps (Table 1).

The ability to detect recombinant proteins using this novel blotting technique takes advantage of enzyme fragment complementation (EFC), whereby a short peptide (the $\alpha$ fragment or enzyme donor) derived from the $\mathrm{N}$ terminus of $\beta$-galactosidase $(\beta$-gal) can reconstitute enzyme activity in a corresponding deletion mutant of full-length $\beta$-gal (the enzyme acceptor or EA) (4). Variants of the $\alpha$ fragment optimized for recombinant expression and high complemented enzyme activity have been created and are known as ProLabel ${ }^{\mathrm{TM}}$ (PL; SSNSLAVVLQRRDWENPGVTQLN RLAAHPPFASWRNSEEARTDRPS QQLRSLNGE; DiscoveRx, Fremont, CA, USA) (5). Since EA essentially lacks $\beta$-gal activity in the absence of PL, EFC technology is ideally suited for use in homogenous assays that can specifically and quantitatively measure PL availability for complementation (5-7). The novel blot assay extends this technology to the detection of recombinant proteins on a solid support, such as a nitrocellulose or polyvinylidine difloride (PDVF) membrane.

To demonstrate the utility of the EFC blot assay, we examined its ability to detect recombinant proteins in extracts prepared from $\mathrm{CHO}$ cells transfected with a variety of PL-tagged constructs. These examples include a stably transfected cell line expressing the nuclear factor of activated $\mathrm{T}$ cells protein tagged at its $\mathrm{C}$ terminus with ProLabel (NFAT-PL) $(8,9)$ as well as transiently transfected cells expressing c-JunPL and cyclin D-PL fusion proteins. Proteins extracted from cell lysates were separated by sodium dodecyl sulfate polyacrylamide gel electrophoresis (SDS-PAGE) and transferred to a nitrocellulose membrane using standard techniques. An additional fusion protein consisting of NeutrAvidin TM chemically conjugated to PL (NA-PL) was included as a control. The blots were incubated for $1 \mathrm{~h}$ with EA reagent (DiscoveRx) to enable complementation with the membrane-bound PL. Chemiluminescent $\beta$-gal substrate (DiscoveRx) was then added directly to the EA solution in which the blot was immersed. Since noncomplemented EA is inactive, it is not necessary to remove or wash excess EA from the membrane. After a 15-min incubation, the blots were drained of excess liquid, placed between sheets of clear plastic, and the $\beta$-gal chemiluminescent activity was detected using a digital imaging system.

A 5-min exposure produced sufficient signal to detect NFAT-PL in a cell lysate sample equivalent to $4 \mu \mathrm{g}$ total protein (Figure 1). A major band with an apparent molecular weight of 85 $\mathrm{kDa}$ was detected, which is consistent with the expected mass of the NFATPL fusion protein $(86.5 \mathrm{kDa})$. Two minor bands were also detected: one of approximately $60 \mathrm{kDa}$ is consistent with it being a proteolytic cleavage product of full-length NFAT-PL, and the other of approximately $6 \mathrm{kDa}$ is consistent with it being the PL peptide moiety (55 amino acids, approximately $6.3 \mathrm{kDa}$ ) liberated by proteolytic cleavage. Transiently expressed c-JunPL and cyclin D-PL of approximately 45 and $42 \mathrm{kDa}$ were detected in cell lysates equivalent to 0.5 and $0.35 \mu \mathrm{g}$ total protein, respectively. Control cell extracts from $\mathrm{CHO}$ cells transiently transfected with vector only lacked 
all bands. The NA-PL control sample exhibited three major bands: the 22 and $27 \mathrm{kDa}$ bands are consistent with the expected molecular weights of a neutravidin monomer conjugated with one or two PL peptides, respectively, and the $5-\mathrm{kDa}$ band is consistent with the free unconjugated PL peptide used in the synthesis (47 amino acids, approximately $5.5 \mathrm{kDa}$ ). There is likely to be little limitation in the size of potential fusions detected by EFC blot, since low molecular weight PL is readily detected in both the NA-PL control and in the inferred cleavage products of other full-length recombinant proteins.

The detection sensitivities of EFC and Western blotting were compared using a protein blot containing a duplicate titration of cell lysate and, consequently, NFAT-PL protein. The blot was divided into two and probed either by EFC as described above or by Western blot analysis, using an antiNFAT monoclonal antibody followed by a horseradish peroxidase (HRP)conjugated secondary antibody and HRP chemiluminescent substrate. Since the samples and transfer conditions were identical for both blots, variation in loading was likely to be minimal. Using identical exposure times to capture chemiluminescent signal, both the EFC and Western blot techniques were able to detect NFAT-PL in lysate containing $0.25 \mu \mathrm{g}$ total protein (Figure 2 ). For the anti-NFAT antibody, pilot titration experiments were required to determine the concentration required to provide sensitivity equivalent to that obtained by EFC blot. While sensitivity was comparable, a higher background was associated with the Western blot compared with the EFC blot. This was primarily due to nonspecific interactions of the primary antibody at the high concentrations used. Similar results were seen in side-by-side EFC/Western blot comparisons with a number of other PL-tagged fusion proteins and a variety of monoclonal and polyclonal antibodies (data not shown). This experiment also demonstrates that EFC blotting is entirely exchangeable with the current protocols for Western blotting, since both methods use the same gel electrophoresis and blotting steps.

EFC blot reagents have a shelf life of at least 6 months and are

Table 1. Comparison of EFC and Western Blot Protocols

\begin{tabular}{|c|c|c|}
\hline $\begin{array}{c}\text { Time } \\
\text { (h) }\end{array}$ & EFC Blot & Western Blot \\
\hline 1 & & \\
\hline & \multicolumn{2}{|c|}{ SDS-PAGE of PL-tagged protein sample and transfer to blot } \\
\hline 2 & & \\
\hline \multicolumn{3}{|c|}{ Brief rinse with water } \\
\hline 3 & $\begin{array}{l}\text { Add } 5 \mathrm{~mL} \text { chemiluminescent } \\
\text { substrate and incubate }(15 \mathrm{~min})\end{array}$ & Incubate with blocking buffer \\
\hline \multirow[t]{2}{*}{4} & $\begin{array}{l}\text { Add } 5 \mathrm{~mL} \text { chemiluminescent } \\
\text { substrate and incubate }(15 \mathrm{~min})\end{array}$ & Incubate with the primary antibody \\
\hline & Acquire image (5-20 $\mathrm{min})$ & \\
\hline 5 & & Wash six times for 10 min each \\
\hline 6 & & Incubate with the secondary antibody \\
\hline 7 & & Wash six times for 10 min each \\
\hline \multirow{2}{*}{8} & & Incubate with detection reagent (5 $\mathrm{min})$ \\
\hline & & Acquire image (5-60 min) \\
\hline $\begin{array}{l}\mathrm{EFC}, \mathrm{e} \\
\text { electro }\end{array}$ & $\begin{array}{l}\text { me fragment complementation; SDS } \\
\text { resis; PL, ProLabel; EA, enzyme acc }\end{array}$ & E, sodium dodecyl sulfate polyacrylamide gel \\
\hline
\end{tabular}

equivalent in cost to conventional Western blot reagents. While the assay has been optimized for use with the substrate provided in the kit (commercialized as the EAstern $^{\mathrm{TM}}$ blot assay; DiscoveRx), it is possible to use other $\beta$-gal substrates, although assay sensitivity and exposure times may vary considerably. In experiments where loading controls are required, a number of options are available that are compatible with EFC blots. Reversible

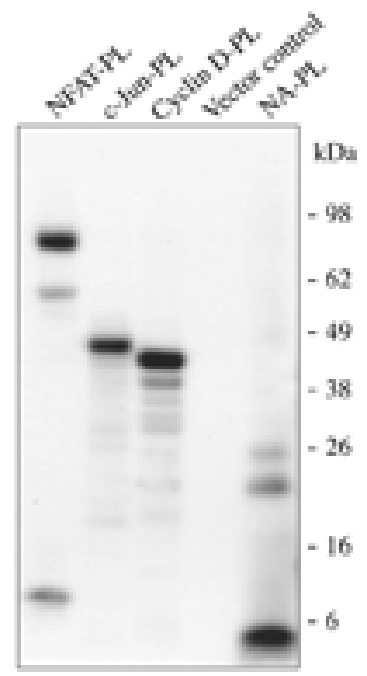

Figure 1. Enzyme fragment complementation (EFC) blot detection of ProLabel (PL)tagged fusion proteins in cell lysates. $\mathrm{CHO}-\mathrm{K} 1$ cells stably transfected with DNA encoding the nuclear factor of activated $\mathrm{T}$ cells protein tagged at its $\mathrm{C}$ terminus with ProLabel (NFAT-PL) were lysed at $4 \times 10^{6}$ cells $/ \mathrm{mL}$ in cell lysis buffer [phosphate-buffered saline (PBS), 0.5\% CHAPS, $1 \times$ Complete Mini EDTA-Free Protease Inhibitor cocktail (Roche Diagnostics, Indianapolis, IN, USA)]. Lysates of CHO-KI cells transiently transfected for 2 days with DNA encoding cJun-PL, cyclin D-PL, and vector only were also generated. Four micrograms NFAT-PL and 0.5 $\mu \mathrm{g}$ c-Jun-PL, $0.35 \mu \mathrm{g}$ cyclin D-PL, or $0.5 \mu \mathrm{g}$ CHO-KI lysates were loaded onto a $4 \%-20 \%$ sodium dodecyl sulfate (SDS) polyacrylamide gel, separated by electrophoresis in MES buffer, and transferred to a nitrocellulose membrane. NAPL control protein was run in an adjacent well. Detergent and other components were removed from the resulting blot by rinsing in ultrapure water for $2 \mathrm{~min}$. The blot was incubated with $5 \mathrm{~mL}$ enzyme acceptor (EA) reagent for $1 \mathrm{~h}$ at room temperature with rocking. Five milliliters chemiluminescent substrate were added, and the blot was incubated for a further $15 \mathrm{~min}$. Excess liquid was drained, and the blot was placed between two sheets of clear plastic. The signal resulting from the complementation of EA with membranebound PL was detected using an Epi Chem II Station digital imaging system (UVP, Upland, CA, USA). Data shown is an inverted image acquired with a 5-min exposure. 
protein stains, such as Ponceau S, can be used to compare total protein levels on a blot prior to EFC analysis. More rigorous analysis such as determination of actin or tubulin levels would require Western blotting. In our experience, a blot in which EFC analysis has been performed can be washed to remove the chemiluminescent substrate and used directly for Western analysis. Blots can also be stored at $4^{\circ} \mathrm{C}$ in phosphatebuffered saline (PBS) prior to EFC or Western blot analysis if necessary.

EFC blotting has several advantages over Western blotting. First, EFC blotting does not require a specific antibody and is therefore

NFAT-PL Lysate ( $\mu \mathrm{g}$ total protein loaded)

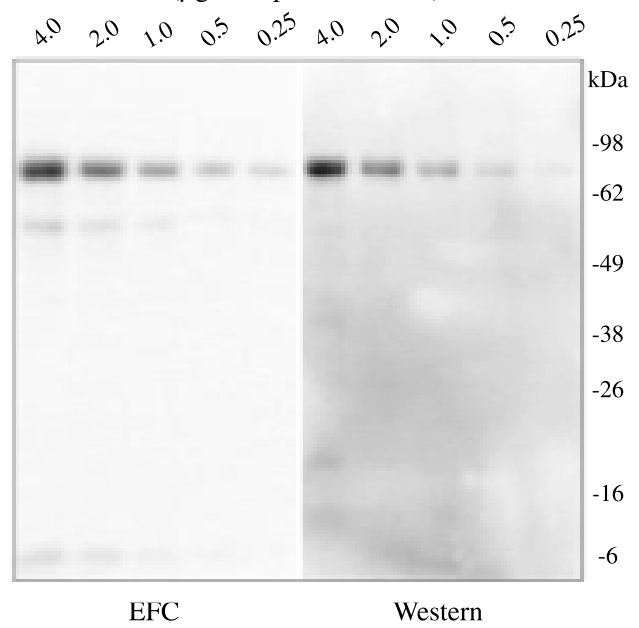

Figure 2. Comparison of enzyme fragment complementation (EFC) and Western blot detection sensitivities. The nuclear factor of activated $\mathrm{T}$ cells protein tagged at its C terminus with ProLabel (NFAT-PL) cell lysate was serially diluted in lysis buffer, separated by sodium dodecyl sulfate polyacrylamide gel electrophoresis (SDS-PAGE), and transferred to a single nitrocellulose membrane as described in Figure 1. The blot, containing duplicate samples sets, was divided in two and probed separately for PL and NFAT using EFC or Western methods, respectively. EFC analysis was performed as described in Figure 1. For Western analysis, the blot was rinsed with water for 2 min, and nonspecific sites were blocked by incubation for 1 h with SuperBlock ${ }^{\circledR}$ (Pierce Biotechnology, Rockford, IL, USA) containing $0.05 \%$ Tween ${ }^{\circledR} 20$. The blot was then incubated for $1 \mathrm{~h}$ with mouse anti-NFAT monoclonal antibody (Cell Signaling Technology, Danvers, MA, USA) diluted 1:1000 in blocking buffer, after which it was washed extensively ( phosphate-buffered saline (PBS) with $0.05 \%$ Tween 20. The blot was then incubated for $1 \mathrm{~h}$ with a goat anti-mouse immunoglobulin $\mathrm{G}$ (IgG)-horseradish peroxidase (HRP) conjugate (Pierce Biotechnology) diluted 1:1000 in blocking buffer, followed by further washing. Five milliliters SuperSignal ${ }^{\circledR}$ West Dura substrate (Pierce Biotechnology) were added to the blot and incubated for $5 \mathrm{~min}$. Signal detection was as described in Figure 1. not dependent on the affinity of the antibody for the protein in question. Thus, in some cases, EFC blotting may provide greater sensitivity, particularly when high affinity antibodies are not available. Second, since EFC blotting is based on enzyme fragment complementation, there is virtually none of the problematic background often associated with Western blotting that can be caused by binding of the primary and/or secondary antibodies to nonspecific proteins and to the blotting membrane itself. Third, the EFC blot has the potential of identifying modifications that alter the apparent molecular mass of the protein, such as phosphorylation, ubiquitination, and glycosylation. While antibodies can be used to measure such events, they could be sensitive to epitope modification. Fourth, EFC blotting provides a significant reduction in the amount of time and number of steps required to detect proteins following transfer from the gel to the blot. In the comparison above (Figure 2), the EFC blot was completed within $1.5 \mathrm{~h}$ and required only two reagent additions and one wash step. In contrast, the Western blot required $4 \mathrm{~h}$ with multiple reagent additions and wash steps. The main limitation of the EFC blot is that it cannot be used to detect endogenous proteins in cells and tissue. In certain situations, such as when loading controls can be performed simultaneously with target detection in Western blot analysis, an EFC blot would be less efficient. For routine analysis, however, EFC blotting offers higher throughput and a simpler protocol without compromising sensitivity. The approach can be applied to the detection of PL-tagged proteins from whole cell lysates as well as affinity purified and immunoprecipitated samples. Finally, protein surveillance using PL and EFC offers a unique and powerful advantage over antibody-based methods that extends beyond protein detection on blots, namely the ability to use the EFC homogenous assay system to rapidly quantify recom- binant protein levels at any point during protein expression and purification.

\section{COMPETING INTERESTS STATEMENT}

The authors are employed by DiscoveRx Corporation, which will market the assay as a commercial product.

\section{REFERENCES}

1. Kurien, B.T. and R.H. Scofield. 2003. Protein blotting: a review. J. Immunol. Methods 274:1-15.

2. McIlhinney, R.A. 2004. Generation and use of epitope-tagged receptors. Methods Mol. Biol. 259:81-98.

3. Patton, W.F. 2002. Detection technologies in proteome analysis. J. Chromatogr. B Analyt. Technol. Biomed. Life Sci. 771:3-31.

4. Eglen, R.M. 2002. Enzyme fragment complementation: a flexible high throughput screening assay technology. Assay Drug Dev. Technol. 1:97-104.

5. Zhao, X., I. Vainshtein, R. Gellibolian, Y. Shu, H. Dotimas, X.M. Wang, P. Fung, J. Horecka, et al. 2003. Homogeneous assays for cellular protein degradation using betagalactosidase complementation: NF-kappaB/ IkappaB pathway signaling. Assay Drug Dev. Technol. 1:823-833.

6. Vainshtein, I., S. Silveria, P. Kaul, R. Rouhani, R.M. Eglen, and J. Wang. 2002. A high-throughput, nonisotopic, competitive binding assay for kinases using nonselective inhibitor probes (ED-NSIP). J. Biomol. Screen. 7:507-514

7. Naqvi, T., A. Lim, R. Rouhani, R. Singh, and R.M. Eglen. 2004. Beta galactosidase enzyme fragment complementation as a highthroughput screening protease technology. J Biomol. Screen. 9:398-408.

8. Macian, F. 2005. NFAT proteins: key regulators of T-cell development and function. Nat. Rev. Immunol. 5:472-484.

9. Horsley, V. and G.K. Pavlath. 2002. NFAT: ubiquitous regulator of cell differentiation and adaptation. J. Cell Biol. 156:771-774.

Address correspondence to Neil W. Charter, DiscoveRx Corporation, 42501 Albrae St., Fremont, CA 94538, USA.e-mail: ncharter@discoverx.com

To purchase reprints of this article, contact

Reprints@BioTechniques.com 